\title{
MULTIPLIERS AND INTEGRATION OPERATORS BETWEEN CONFORMALLY INVARIANT SPACES
}

\author{
DANIEL GIRELA AND NOEL MERCHÁN
}

\begin{abstract}
In this paper we are concerned with two classes of conformally invariant spaces of analytic functions in the unit disc $\mathbb{D}$, the Besov spaces $B^{p}(1 \leq p<\infty)$ and the $Q_{s}$ spaces $(0<s<\infty)$. Our main objective is to characterize for a given pair $(X, Y)$ of spaces in these classes, the space of pointwise multipliers $M(X, Y)$, as well as to study the related questions of obtaining characterizations of those $g$ analytic in $\mathbb{D}$ such that the Volterra operator $T_{g}$ or the companion operator $I_{g}$ with symbol $g$ is a bounded operator from $X$ into $Y$.
\end{abstract}

\section{INTRODUCTION}

Let $\mathbb{D}=\{z \in \mathbb{C}:|z|<1\}$ denote the open unit disc of the complex plane $\mathbb{C}$ and let $\mathcal{H o l}(\mathbb{D})$ be the space of all analytic functions in $\mathbb{D}$ endowed with the topology of uniform convergence on compact subsets.

If $0<r<1$ and $f \in \mathcal{H} o l(\mathbb{D})$, we set

$$
\begin{aligned}
M_{p}(r, f) & =\left(\frac{1}{2 \pi} \int_{0}^{2 \pi}\left|f\left(r e^{i t}\right)\right|^{p} d t\right)^{1 / p}, \quad 0<p<\infty \\
M_{\infty}(r, f) & =\sup _{|z|=r}|f(z)| .
\end{aligned}
$$

If $0<p \leq \infty$ the Hardy space $H^{p}$ consists of those $f \in \mathcal{H o l}(\mathbb{D})$ such that

$$
\|f\|_{H^{p}} \stackrel{\text { def }}{=} \sup _{0<r<1} M_{p}(r, f)<\infty .
$$

We mention [18] for the theory of $H^{p}$-spaces.

If $0<p<\infty$ and $\alpha>-1$, the weighted Bergman space $A_{\alpha}^{p}$ consists of those $f \in \mathcal{H o l}(\mathbb{D})$ such that

$$
\|f\|_{A_{\alpha}^{p}} \stackrel{\text { def }}{=}\left((\alpha+1) \int_{\mathbb{D}}(1-|z|)^{\alpha}|f(z)|^{p} d A(z)\right)^{1 / p}<\infty .
$$

The unweighted Bergman space $A_{0}^{p}$ is simply denoted by $A^{p}$. Here, $d A(z)=$ $\frac{1}{\pi} d x d y$ denotes the normalized Lebesgue area measure in $\mathbb{D}$. We refer to [19], [36] and [58] for the theory of these spaces.

2010 Mathematics Subject Classification. 30H25, 47B38.

Key words and phrases. Möbius invariant spaces and Besov spaces and $Q_{s}$ spaces and multipliers and integration operators and Carleson measures.

This research is supported in part by a grant from "El Ministerio de Ciencia, Innovación y Universidades", Spain (PGC2018-096166-B-I00) and by grants from la Junta de Andalucía (FQM-210 and UMA18-FEDERJA-002). 
We let $\operatorname{Aut}(\mathbb{D})$ denote the set of all disc automorphisms, that is, of all one-toone analytic maps $\varphi$ from $\mathbb{D}$ onto itself. It is well known that Aut $(\mathbb{D})$ coincides with the set of all Möbius transformations from $\mathbb{D}$ onto itself:

$$
\operatorname{Aut}(\mathbb{D})=\left\{\lambda \varphi_{a}:|\lambda|=1, a \in \mathbb{D}\right\},
$$

where $\varphi_{a}(z)=(a-z) /(1-\bar{a} z)(z \in \mathbb{D})$.

A linear space $X$ of analytic functions in $\mathbb{D}$ is said to be conformally invariant or Möbius invariant if whenever $f \in X$, then also $f \circ \varphi \in X$ for any $\varphi \in \operatorname{Aut}(\mathbb{D})$ and, moreover, $X$ is equipped with a semi-norm $\rho$ for which there exists a positive constant $C$ such that

$$
\rho(f \circ \varphi) \leq C \rho(f), \quad \text { whenever } f \in X \text { and } \varphi \in \operatorname{Aut}(\mathbb{D}) .
$$

The articles [8] and [44] are fundamental references for the theory of Möbius invariant spaces which have attracted much attention in recent years (see, e.g., $[3,16,17,30,47,57,58])$.

The Bloch space $\mathcal{B}$ consists of all analytic functions $f$ in $\mathbb{D}$ such that

$$
\rho_{\mathcal{B}}(f) \stackrel{\text { def }}{=} \sup _{z \in \mathbb{D}}\left(1-|z|^{2}\right)\left|f^{\prime}(z)\right|<\infty \text {. }
$$

The Schwarz-Pick lemma easily implies that $\rho_{\mathcal{B}}$ is a conformally invariant seminorm, thus $\mathcal{B}$ is a conformally invariant space. It is also a Banach space with the norm $\|\cdot\|_{\mathcal{B}}$ defined by $\|f\|_{\mathcal{B}}=|f(0)|+\rho_{\mathcal{B}}(f)$. The little Bloch space $\mathcal{B}_{0}$ is the set of those $f \in \mathcal{B}$ such that $\lim _{|z| \rightarrow 1}\left(1-|z|^{2}\right)\left|f^{\prime}(z)\right|=0$. Alternatively, $\mathcal{B}_{0}$ is the closure of the polynomials in the Bloch norm. A classical reference for the theory of Bloch functions is [7]. Rubel and Timoney [44] proved that $\mathcal{B}$ is the largest "reasonable" Möbius invariant space. More precisely, they proved the following result.

Theorem A. Let $X$ be a Möbius invariant linear space of analytic functions in $\mathbb{D}$ and let $\rho$ be a Möbius invariant seminorm on $X$. If there exists a non-zero decent linear functional $L$ on $X$ which is continuous with respect to $\rho$, then $X \subset \mathcal{B}$ and there exists a constant $A>0$ such that $\rho_{\mathcal{B}}(f) \leq A \rho(f)$, for all $f \in X$.

Here, a linear functional $L$ on $X$ is said to be decent if it extends continuously to $\mathcal{H o l}(\mathbb{D})$.

The space $B M O A$ consists of those functions $f$ in $H^{1}$ whose boundary values have bounded mean oscillation on the unit circle $\partial \mathbb{D}$ as defined by $F$. John and L. Nirenberg. There are many characterizations of $B M O A$ functions. Let us mention the following:

If $f \in \mathcal{H o l}(\mathbb{D})$, then $f \in B M O A$ if and only if $\|f\|_{B M O A} \stackrel{\text { def }}{=}|f(0)|+\rho_{*}(f)<\infty$, where

$$
\rho_{*}(f)=\sup _{a \in \mathbb{D}}\left\|f \circ \varphi_{a}-f(a)\right\|_{H^{2}} .
$$

It is well known that $H^{\infty} \subset B M O A \subset \mathcal{B}$ and that $B M O A$ equipped with the seminorm $\rho_{*}$ is a Möbius invariant space. The space $V M O A$ consists of those $f \in B M O A$ such that $\lim _{|a| \rightarrow 1}\left\|f \circ \varphi_{a}-f(a)\right\|_{H^{2}}=0$, it is the closure of the 
polynomials in the $B M O A$-norm. We mention [28] as a general reference for the space $B M O A$.

Other important Möbius invariant spaces are the Besov spaces and the $Q_{s}$ spaces.

For $1<p<\infty$, the analytic Besov space $B^{p}$ is defined as the set of all functions $f$ analytic in $\mathbb{D}$ such that $f^{\prime} \in A_{p-2}^{p}$. All $B^{p}$ spaces $(1<p<\infty)$ are conformally invariant with respect to the semi-norm $\rho_{B^{p}}$ defined by

$$
\rho_{B^{p}}(f) \stackrel{\text { def }}{=}\left\|f^{\prime}\right\|_{A_{p-2}^{p}}
$$

(see [8, p. 112] or [16, p. 46]) and Banach spaces with the norm $\|\cdot\|_{B^{p}}$ defined by $\|f\|_{B^{p}}=|f(0)|+\rho_{B^{p}}(f)$. An important and well-studied case is the classical Dirichlet space $B^{2}$ (often denoted by $\mathcal{D}$ ) of analytic functions whose image has a finite area, counting multiplicities.

The space $B^{1}$ requires a special definition: it is the space of all analytic functions $f$ in $\mathbb{D}$ for which $f^{\prime \prime} \in A^{1}$. Although the semi-norm $\rho$ defined by $\rho(f)=\left\|f^{\prime \prime}\right\|_{A^{1}}$ is not conformally invariant, the space itself is. An alternative definition of $B^{1}$ with a conformally invariant semi-norm is given in [8], where it is also proved that $B^{1}$ is contained in any Möbius invariant space. A lot of information on Besov spaces can be found in $[8,16,17,37,56,58]$. Let us recall that

$$
\begin{aligned}
& V M O A \subsetneq \mathcal{B}_{0}, \quad B M O A \subsetneq \mathcal{B}, \\
& B^{1} \subsetneq B^{p} \subsetneq B^{q} \subsetneq V M O A \subsetneq B M O A, \quad 1<p<q<\infty .
\end{aligned}
$$

If $0 \leq s<\infty$, we say that $f \in Q_{s}$ if $f$ is analytic in $\mathbb{D}$ and

$$
\sup _{a \in \mathbb{D}} \int_{\mathbb{D}}\left|f^{\prime}(z)\right|^{2} g(z, a)^{s} d A(z)<\infty
$$

where $g(z, a)=\log (|1-\bar{a} z| /|a-z|)$ is the Green function of $\mathbb{D}$. These spaces were introduced by Aulaskari and Lappan [12] while looking for characterizations of Bloch functions (see [50] for the case $s=2$ ). For $s>1, Q_{s}$ is the Bloch space, $Q_{1}=B M O A$, and

$$
\mathcal{D} \subsetneq Q_{s_{1}} \subsetneq Q_{s_{2}} \subsetneq B M O A, \quad 0<s_{1}<s_{2}<1 .
$$

It is well known $[14,46]$ that for every $s$ with $0 \leq s<\infty$, a function $f \in \mathcal{H o l}(\mathbb{D})$ belongs to $Q_{s}$ if and only if

$$
\rho_{Q_{s}}(f) \stackrel{\text { def }}{=}\left(\sup _{a \in \mathbb{D}} \int_{\mathbb{D}}\left|f^{\prime}(z)\right|^{2}\left(1-\left|\varphi_{a}(z)\right|^{2}\right)^{s} d A(z)\right)^{1 / 2}<\infty .
$$

All $Q_{s}$ spaces $(0 \leq s<\infty)$ are conformally invariant with respect to the seminorm $\rho_{Q_{s}}$. They are also Banach spaces with the norm $\|\cdot\|_{Q_{s}}$ defined by $\|f\|_{Q_{s}}=$ $|f(0)|+\rho_{Q_{s}}(f)$. We mention $[52,53]$ as excellent references for the theory of $Q_{s}$-spaces.

Let us recall the following two facts which were first observed in [10].

$$
\text { If } 0<p \leq 2 \text {, then } B^{p} \subset Q_{s} \text { for all } s>0 \text {. }
$$




$$
\text { If } 2<p<\infty \text {, then } B^{p} \subset Q_{s} \text { if and only if } 1-\frac{2}{p}<s \text {. }
$$

For $g$ analytic in $\mathbb{D}$, the Volterra operator $T_{g}$ is defined as follows:

$$
T_{g}(f)(z) \stackrel{\text { def }}{=} \int_{0}^{z} g^{\prime}(\xi) f(\xi) d \xi, \quad f \in \mathcal{H} o l(\mathbb{D}), \quad z \in \mathbb{D} .
$$

We define also the companion operator $I_{g}$ by

$$
I_{g}(f)(z) \stackrel{\text { def }}{=} \int_{0}^{z} g(\xi) f^{\prime}(\xi) d \xi, \quad f \in \mathcal{H} o l(\mathbb{D}), \quad z \in \mathbb{D} .
$$

The integration operators $T_{g}$ and $I_{g}$ have been studied in a good number of papers. Let us just mention here that Pommerenke [43] proved that $T_{g}$ is bounded on $H^{2}$ if and only if $g \in B M O A$ and that Aleman and Siskakis [4] characterized those $g \in \mathcal{H} o l(\mathbb{D})$ for which $T_{g}$ is bounded on $H^{p}(p \geq 1)$, while Aleman and Cima characterized in [1] those $g \in \mathcal{H o l}(\mathbb{D})$ for which $T_{g}$ maps $H^{p}$ into $H^{q}$. Aleman and Siskakis [5] studied the operators $I_{g}$ and $T_{g}$ acting on Bergman spaces.

For $g \in \mathcal{H o l}(\mathbb{D})$, the multiplication operator $M_{g}$ is defined by

$$
M_{g}(f)(z) \stackrel{\text { def }}{=} g(z) f(z), \quad f \in \mathcal{H} \text { ol }(\mathbb{D}), z \in \mathbb{D} .
$$

If $X$ and $Y$ are two Banach spaces of analytic function in $\mathbb{D}$ continuously embedded in $\mathcal{H o l}(\mathbb{D})$ and $g \in \mathcal{H o l}(\mathbb{D})$ then $g$ is said to be a multiplier from $X$ to $Y$ if $M_{g}(X) \subset Y$. The space of all multipliers from $X$ to $Y$ will be denoted by $M(X, Y)$ and $M(X)$ will stand for $M(X, X)$. Using the closed graph theorem we see that for the three operators $T_{g}, I_{g}, M_{g}$, we have that if one of them maps $X$ into $Y$ then it is continuous from $X$ to $Y$. We remark also that

$$
T_{g}(f)+I_{g}(f)=M_{g}(f)-f(0) g(0) .
$$

Thus if two of the operators $T_{g}, I_{g}, M_{g}$ are bounded from $X$ to $Y$ so is the third one.

It is well known that if $X$ is nontrivial then $M(X) \subset H^{\infty}$ (see, e. g., [2, Lemma 1.1] or [48, Lemma 1.10]), but $M(X, Y)$ need not be included in $H^{\infty}$ if $Y \not \subset X$. However, when dealing with Möbius invariant spaces we have the following result.

Proposition 1.1. Let $X$ and $Y$ be two Möbius invariant spaces of analytic functions in $\mathbb{D}$ equipped with the seminorms $\rho_{X}$ and $\rho_{Y}$, respectively. Suppose that there exists a non-trivial decent linear functional $L$ on $Y$ which is continuous with respect to $\rho_{Y}$. Let $g \in \mathcal{H o l}(\mathbb{D})$. Then the following statements hold.

(i) If $M_{g}$ is continuous from $\left(X, \rho_{X}\right)$ into $\left(Y, \rho_{Y}\right)$, then $g \in H^{\infty}$.

(ii) If $I_{g}$ is continuous from $\left(X, \rho_{X}\right)$ into $\left(Y, \rho_{Y}\right)$, then $g \in H^{\infty}$.

Before embarking into the proof of Proposition 1.1, let us mention that, as usual, throughout the paper we shall be using the convention that $C=C(p, \alpha, q, \beta, \ldots)$ will denote a positive constant which depends only upon the displayed parameters $p, \alpha, q, \beta \ldots$ (which sometimes will be omitted) but not necessarily the same at different occurrences. Moreover, for two real-valued functions $E_{1}, E_{2}$ we write 
$E_{1} \lesssim E_{2}$, or $E_{1} \gtrsim E_{2}$, if there exists a positive constant $C$ independent of the arguments such that $E_{1} \leq C E_{2}$, respectively $E_{1} \geq C E_{2}$. If we have $E_{1} \lesssim E_{2}$ and $E_{1} \gtrsim E_{2}$ simultaneously then we say that $E_{1}$ and $E_{2}$ are equivalent and we write $E_{1} \asymp E_{2}$. Also, if $1<p<\infty, p^{\prime}$ will stand for its conjugate exponent, that is, $\frac{1}{p}+\frac{1}{p^{\prime}}=1$.

Proof of Proposition 1.1. Since $X$ is conformally invariant, $\operatorname{Aut}(\mathbb{D}) \subset X[8$, p. 114] and

$$
\rho_{X}\left(\varphi_{a}\right) \asymp 1, \quad a \in \mathbb{D} .
$$

Suppose that $M_{g}$ is continuous from $\left(X, \rho_{X}\right)$ into $\left(Y, \rho_{Y}\right)$. Using this, Theorem $\mathrm{A}$, and (1.4) we obtain

$$
\rho_{\mathcal{B}}\left(g \varphi_{a}\right) \lesssim \rho_{Y}\left(g \varphi_{a}\right) \lesssim \rho_{X}\left(\varphi_{a}\right) \lesssim 1, \quad a \in \mathbb{D} .
$$

This implies that

$$
\left(1-|a|^{2}\right)\left|g^{\prime}(a) \varphi_{a}(a)+g(a) \varphi_{a}^{\prime}(a)\right| \lesssim 1, \quad a \in \mathbb{D} .
$$

Since $\varphi(a)=0$ and $\varphi_{a}^{\prime}(a)=-\left(1-|a|^{2}\right)^{-1}$, it follows that

$$
|g(a)| \lesssim 1, \quad a \in \mathbb{D}
$$

that is, $g \in H^{\infty}$.

Similarly, if we assume that $I_{g}$ is continuous from $\left(X, \rho_{X}\right)$ into $\left(Y, \rho_{Y}\right)$, we obtain

$$
\rho_{\mathcal{B}}\left(I_{g}\left(\varphi_{a}\right)\right) \lesssim 1, \quad a \in \mathbb{D}
$$

This implies that

$$
\left(1-|a|^{2}\right)\left|\left(I_{g}\left(\varphi_{a}\right)\right)^{\prime}(a)\right|=\left(1-|a|^{2}\right)\left|\varphi_{a}^{\prime}(a)\right||g(a)|=|g(a)| \lesssim 1, \quad a \in \mathbb{D} .
$$

For notational convenience, set

$$
\mathcal{B Q}=\left\{Q_{s}: 0 \leq s<\infty\right\} \cup\left\{B^{p}: 1 \leq p<\infty\right\}
$$

The main purpose of this paper is characterizing, for a given pair of spaces $X, Y \in$ $\mathcal{B Q}$, the functions $g \in \mathcal{H o l}(\mathbb{D})$ such that the operators $M_{g}, T_{g}$ and/or $I_{g}$ map $X$ into $Y$. When $X$ and $Y$ are Besov spaces this question has been extensively studied (see, e.g. $[9,26,32,45,49,59]$ ). Thus we shall concentrate ourselves to study these operators when acting between a certain Besov space $B^{p}$ and a certain $Q_{s}$ space and when acting between $Q_{s_{1}}$ and $Q_{s_{2}}$ for a certain pair of positive numbers $s_{1}, s_{2}$.

\section{Multipliers and integration operators From Besov spaCes into

$$
Q_{s} \text {-SPACES }
$$

For $\alpha>0$, the $\alpha$-logarithmic Bloch space $\mathcal{B}_{\log , \alpha}$ is the Banach space of those functions $f \in \mathcal{H o l}(\mathbb{D})$ which satisfy

$$
\|f\|_{\log , \alpha} \stackrel{\text { def }}{=}|f(0)|+\sup _{z \in \mathbb{D}}\left(1-|z|^{2}\right)\left(\log \frac{2}{1-|z|^{2}}\right)^{\alpha}\left|f^{\prime}(z)\right|<\infty .
$$

For simplicity, the space $\mathcal{B}_{\log , 1}$ will be denoted by $\mathcal{B}_{\log }$. 
It is clear that $B_{\log , \alpha} \subset \mathcal{B}_{0}$, for all $\alpha>0$. Using the characterization of $V M O A$ in terms of Carleson measures [28, p. 102], it follows easily that

$$
B_{\log , \alpha} \subset V M O A, \quad \text { for all } \alpha>1 / 2 \text {. }
$$

In particular, $\mathcal{B}_{\log } \subset V M O A$.

Brown and Shields [15] showed that $M(\mathcal{B})=\mathcal{B}_{\log } \cap H^{\infty}$. The spaces $M\left(B^{p}, \mathcal{B}\right)$ $(1 \leq p<\infty)$ were characterized in [25]. Namely, Theorem 1 of [25] asserts that $M\left(B^{1}, \mathcal{B}\right)=H^{\infty}$ and

$$
M\left(B^{p}, \mathcal{B}\right)=H^{\infty} \cap \mathcal{B}_{\log , 1 / p^{\prime}}, \quad 1<p<\infty,
$$

where $p^{\prime}$ is the exponent conjugate to $p$, that is, $\frac{1}{p}+\frac{1}{p^{\prime}}=1$.

In this section we extend these results. In particular, we shall obtain for any pair $(p, s)$ with $2<p<\infty$ and $0<s<\infty$ a complete characterization of the space of multipliers $M\left(B^{p}, Q_{s}\right)$.

Let us start with the case $s \geq 1$ which is the simplest one.

Theorem 2.1. Let $g \in \mathcal{H}$ ol $(\mathbb{D})$. Then:

(i) $I_{g}$ maps $B^{1}$ into $\mathcal{B}$ if and only if $g \in H^{\infty}$.

(ii) $M_{g}$ maps $B^{1}$ into $\mathcal{B}$ if and only if $g \in H^{\infty}$.

(iii) $T_{g}$ maps $B^{1}$ into $\mathcal{B}$ if and only if $g \in \mathcal{B}$.

Proof. If $I_{g}\left(B^{1}\right) \subset \mathcal{B}$ then, using Proposition 1.1, it follows that $g \in H^{\infty}$.

To prove the converse it suffices to recall that $B^{1} \subset \mathcal{B}$. Indeed, suppose that $g \in H^{\infty}$ and take $f \in B^{1}$. Then

$$
\left(1-|z|^{2}\right)\left|\left(I_{g}(f)\right)^{\prime}(z)\right|=\left(1-|z|^{2}\right)\left|f^{\prime}(z)\|g(z) \mid \leq\| f\left\|_{\mathcal{B}}\right\| g \|_{H^{\infty}} .\right.
$$

Thus $I_{g}(f) \in \mathcal{B}$.

Hence (i) is proved. Now, (ii) is contained in [25, Theorem 1].

It remains to prove (iii). If $T_{g}\left(B^{1}\right) \subset \mathcal{B}$ then $T_{g}(1)=g-g(0) \in \mathcal{B}$ and, hence $g \in \mathcal{B}$. Conversely, if $g \in \mathcal{B}$ and $f \in \mathcal{B}^{1}$ then, using the fact that $B^{1} \subset H^{\infty}$, we obtain

$$
\left(1-|z|^{2}\right)\left|\left(T_{g}(f)\right)^{\prime}(z)\right|=\left(1-|z|^{2}\right)\left|g^{\prime}(z)\right||f(z)| \leq\|g\|_{\mathcal{B}}\|f\|_{H^{\infty}} .
$$

Thus $T_{g}(f) \in \mathcal{B}$. Hence (iii) is also proved.

Theorem 2.2. Suppose that $1<p<\infty, \frac{1}{p}+\frac{1}{p^{\prime}}=1$, and let $g \in \mathcal{H}$ ol $(\mathbb{D})$. Then:

(i) $I_{g}$ maps $B^{p}$ into $\mathcal{B}$ if and only if $g \in H^{\infty}$.

(ii) $M_{g}$ maps $B^{p}$ into $\mathcal{B}$ if and only if $g \in H^{\infty} \cap \mathcal{B}_{\log , 1 / p^{\prime}}$.

(iii) $T_{g}$ maps $B^{p}$ into $\mathcal{B}$ if and only if $g \in \mathcal{B}_{\log , 1 / p^{\prime}}$.

Proof. If $I_{g}$ maps $B^{p}$ into $\mathcal{B}$ then Proposition 1.1 implies that $g \in H^{\infty}$. Conversely, using that $B^{p} \subset \mathcal{B}$, we see that if $g \in H^{\infty}$ and $f \in B^{p}$ then

$$
\left(1-|z|^{2}\right)\left|\left(I_{g}(f)\right)^{\prime}(z)\right|=\left(1-|z|^{2}\right)\left|f^{\prime}(z)\right||g(z)| \leq\|f\|_{\mathcal{B}}\|g\|_{H^{\infty}} .
$$

Hence, $I_{g}(f) \in \mathcal{B}$. Thus (i) is proved and (ii) reduces to (2.2).

Finally, (iii) follows from the following more precise result. 
Theorem 2.3. Suppose that $1<p<\infty, \frac{1}{p}+\frac{1}{p^{\prime}}=1$, and let $g \in \mathcal{H o l}(\mathbb{D})$. Then the following conditions are equivalent.

(a) $T_{g}$ maps $B^{p}$ into $\mathcal{B}$.

(b) $g \in \mathcal{B}_{\log , 1 / p^{\prime}}$

(c) $T_{g}$ maps $B^{p}$ into $\mathcal{B}_{0}$.

Proof of Theorem 2.3. (a) $\Rightarrow$ (b) Suppose (a). By the closed graph theorem $T_{g}$ is a bounded operator from $B^{p}$ into $\mathcal{B}$, hence

$$
\left(1-|z|^{2}\right)\left|g^{\prime}(z) f(z)\right| \lesssim\|f\|_{B^{p}}, \quad z \in \mathbb{D}, f \in B^{p} .
$$

For $a \in \mathbb{D}$ with $a \neq 0$, set

$$
f_{a}(z)=\left(\log \frac{1}{1-|a|^{2}}\right)^{-1 / p} \log \frac{1}{1-\bar{a} z}, \quad z \in \mathbb{D} .
$$

It is readily seen that $f_{a} \in B^{p}$ for all $a$ and that $\left\|f_{a}\right\|_{B^{p}} \asymp 1$. Using this and taking $f=f_{a}$ and $z=a$ in (2.3), we obtain

$$
\left(1-|a|^{2}\right)\left|g^{\prime}(a)\right|\left(\frac{1}{\left.1-|a|^{2}\right)}\right)^{1 / p^{\prime}} \lesssim 1
$$

that is $g \in \mathcal{B}_{\log , 1 / p^{\prime}}$.

(b) $\Rightarrow(\mathrm{c})$ Suppose (b) and take $f \in B^{p}$. It is well known that

$$
|f(z)|=\mathrm{o}\left(\left(\log \frac{1}{1-|z|^{2}}\right)^{1 / p^{\prime}}\right), \quad \text { as }|z| \rightarrow 1,
$$

(see, e.g., [37, 56]). This and (b) immediately yield that $T_{g}(f) \in \mathcal{B}_{0}$.

The implication (c) $\Rightarrow$ (a) is trivial. Hence the proof of Theorem 2.3 is finished and, consequently, Theorem 2.2 is also proved.

Let us turn now to the case $0<s \leq 1$. We shall consider first the Volterra operators $T_{g}$. For $0<s<\infty$ and $\alpha>0$ we set

$Q_{s, \log , \alpha}=\left\{f \in \mathcal{H}\right.$ ol $\left.(\mathbb{D}): \sup _{a \in \mathbb{D}}\left(\log \frac{2}{1-|a|}\right)^{2 \alpha} \int_{\mathbb{D}}\left|f^{\prime}(z)\right|^{2}\left(1-\left|\varphi_{a}(z)\right|^{2}\right)^{s} d A(z)<\infty\right\}$.

We have the following results.

Theorem 2.4. Suppose that $0<s \leq 1$ and let $g \in \mathcal{H o l}(\mathbb{D})$. Then:

(i) $T_{g}$ maps $B^{1}$ into $Q_{s}$ if and only if $g \in Q_{s}$.

(ii) If $1<p<\infty, 0<s \leq 1$, and $T_{g}$ maps $B^{p}$ into $Q_{s}$, then $g \in Q_{s, \log , 1 / p^{\prime}}$.

(iii) If $1<p<\infty$, then $T_{g}$ maps $B^{p}$ into $Q_{1}=B M O A$ if and only if $g \in$ $Q_{1, \log , 1 / p^{\prime}}$

(iv) If $2<p<\infty, 0<s<1$, and $1-\frac{2}{p}<s$ then $T_{g}$ maps $B^{p}$ into $Q_{s}$ if and only if $g \in Q_{s, \log , 1 / p^{\prime}}$.

Before we get into the proofs of these results we shall introduce some notation and recall some results which will be needed in our work. 
If $I \subset \partial \mathbb{D}$ is an interval, $|I|$ will denote the length of $I$. The Carleson square $S(I)$ is defined as $S(I)=\left\{r e^{i t}: e^{i t} \in I, \quad 1-\frac{|I|}{2 \pi} \leq r<1\right\}$. Also, for $a \in \mathbb{D}$, the Carleson box $S(a)$ is defined by

$$
S(a)=\left\{z \in \mathbb{D}: 1-|z| \leq 1-|a|,\left|\frac{\arg (a \bar{z})}{2 \pi}\right| \leq \frac{1-|a|}{2}\right\} .
$$

If $s>0$ and $\mu$ is a positive Borel measure on $\mathbb{D}$, we shall say that $\mu$ is an $s$-Carleson measure if there exists a positive constant $C$ such that

$$
\mu(S(I)) \leq C|I|^{s}, \quad \text { for any interval } I \subset \partial \mathbb{D},
$$

or, equivalently, if there exists $C>0$ such that

$$
\mu(S(a)) \leq C(1-|a|)^{s}, \quad \text { for all } a \in \mathbb{D} .
$$

A 1-Carleson measure will be simply called a Carleson measure.

These concepts were generalized in [55] as follows: If $\mu$ is a positive Borel measure in $\mathbb{D}, 0 \leq \alpha<\infty$, and $0<s<\infty$, we say that $\mu$ is an $\alpha$-logarithmic $s$-Carleson measure if there exists a positive constant $C$ such that

$$
\frac{\mu(S(I))\left(\log \frac{2 \pi}{|I|}\right)^{\alpha}}{|I|^{s}} \leq C, \quad \text { for any interval } I \subset \partial \mathbb{D}
$$

or, equivalently, if

$$
\sup _{a \in \mathbb{D}} \frac{\mu(S(a))\left(\log \frac{2}{1-|a|^{2}}\right)^{\alpha}}{\left(1-|a|^{2}\right)^{s}}<\infty .
$$

Carleson measures and logarithmic Carleson measures are known to play a basic role in the study of the boundedness of a great number of operators between analytic function spaces. In particular we recall the Carleson embedding theorem for Hardy spaces which asserts that if $0<p<\infty$ and $\mu$ is a positive Borel measure on $\mathbb{D}$ then $\mu$ is a Carleson measure if and only if the Hardy space $H^{p}$ is continuously embedded in $L^{p}(d \mu)$ (see [18, Chapter 9]).

In the next theorem we collect a number of known results which will be needed in our work.

Theorem B. (i) If $0<s \leq 1$ and $f \in \mathcal{H}$ ol $(\mathbb{D})$, then $f \in Q_{s}$ if and only if the Borel measure $\mu$ on $D$ defined by

$$
d \mu(z)=\left(1-|z|^{2}\right)^{s}\left|f^{\prime}(z)\right|^{2} d A(z)
$$

is an $s$-Carleson measure.

(ii) If $0 \leq \alpha<\infty, 0<s<\infty$, and $\mu$ is a positive Borel measure on $\mathbb{D}$ then $\mu$ is an $\alpha$-logarithmic s-Carleson measure if and only if

$$
\sup _{a \in \mathbb{D}}\left(\log \frac{2}{1-|a|^{2}}\right)^{\alpha} \int_{\mathbb{D}}\left(\frac{1-|a|^{2}}{|1-\bar{a} z|^{2}}\right)^{s} d \mu(z)<\infty .
$$

(iii) If $1<p \leq 2$ then $B^{p} \subset Q_{s}$ for all $s>0$.

(iv) If $2<p<\infty$ and $1-\frac{2}{p}<s$, then $B^{p} \subset Q_{s}$. 
(v) For $s>-1$, we let $\mathcal{D}_{s}$ be the space of those functions $f \in \mathcal{H}$ ol $(\mathbb{D})$ for which

$$
\|f\|_{\mathcal{D}_{s}} \stackrel{\text { def }}{=}|f(0)|+\left(\int_{\mathbb{D}}\left(1-|z|^{2}\right)^{s}\left|f^{\prime}(z)\right|^{2} d A(z)\right)^{1 / 2}<\infty .
$$

Suppose that $0<s<1$ and $\alpha>1$, and let $\mu$ be a positive Borel measure on $\mathbb{D}$. If $\mu$ is an $\alpha$-logarithmic $s$ Carleson measure, then $\mu$ is a Carleson measures for $\mathcal{D}_{s}$, that is, $\mathcal{D}_{s}$ is continuously embedded in $L^{2}(d \mu)$.

Let us mention that (i) is due to Aulaskari, Stegenga and Xiao [13], (ii) is due to Zhao [55], (iii) and (iv) were proved by Aulaskari and Csordas in [10], and (v) is due to Pau and Peláez [41, Lemma 1].

Using Theorem B (ii) and the fact that

$$
1-|\varphi(z)|^{2}=\frac{\left(1-|a|^{2}\right)\left(1-|z|^{2}\right)}{|1-\bar{a} z|^{2}}
$$

we see that for a function $f \in \mathcal{H o l}(\mathbb{D})$ we have that $f \in Q_{s, \log , \alpha}$ if and only if the measure $\mu$ defined by $d \mu(z)=\left(1-|z|^{2}\right)^{s}\left|f^{\prime}(z)\right|^{2} d A(z)$ is a $2 \alpha$-logarithmic $s$-Carleson measure.

Proof of Theorem 2.4 (i). Suppose that $T_{g}$ maps $B^{1}$ into $Q_{s}$. Since the constant functions belong to $B^{1}$, we have that $T_{g}(1)=g-g(0) \in Q_{s}$ and, hence, $g \in Q_{s}$.

To prove the converse, suppose that $g \in Q_{s}$. Then the measure $\mu$ defined by

$$
d \mu(z)=\left(1-|z|^{2}\right)^{s}\left|g^{\prime}(z)\right|^{2} d A(z)
$$

is an $s$-Carleson measure. Take now $f \in B^{1}$, then $f \in H^{\infty}$ and, hence,

$$
\left(1-|z|^{2}\right)^{s}\left|\left(T_{g}(f)\right)^{\prime}(z)\right|^{2}=\left(1-|z|^{2}\right)^{s}\left|g^{\prime}(z)\right|^{2}|f(z)|^{2} \leq\|f\|_{H^{\infty}}^{2}\left(1-|z|^{2}\right)^{s}\left|g^{\prime}(z)\right|^{2} \text {. }
$$

Since $\mu$ is an $s$-Carleson measure, it follows readily that the measure $\nu$ given by $d \nu(z)=\left(1-|z|^{2}\right)^{s}\left|\left(T_{g}(f)\right)^{\prime}(z)\right|^{2} d A(z)$ is also an $s$-Carleson measure and, hence, $T_{g}(f) \in Q_{s}$.

Proof of Theorem 2.4 (ii).

Suppose that $0<s \leq 1,1<p<\infty$, and that $T_{g}$ maps $B^{p}$ into $Q_{s}$. For $a \in \mathbb{D} \backslash\{0\}$, set

$$
f_{a}(z)=\left(\log \frac{1}{1-|a|^{2}}\right)^{-1 / p} \log \frac{1}{1-\bar{a} z}, \quad z \in \mathbb{D},
$$

as in (2.4). We have that $\left\|f_{a}\right\|_{B^{p}} \asymp 1$ and it is also clear that

$$
\left|f_{a}(z)\right| \asymp\left(\log \frac{1}{1-|a|^{2}}\right)^{1 / p^{\prime}}, \quad z \in S(a) .
$$


Using these facts, we obtain

$$
\begin{aligned}
& \frac{\left(\log \frac{1}{1-|a|^{2}}\right)^{2 / p^{\prime}}}{\left(1-|a|^{2}\right)^{s}} \int_{S(a)}\left(1-|z|^{2}\right)^{s}\left|g^{\prime}(z)\right|^{2} d A(z) \\
\asymp & \frac{1}{\left(1-|a|^{2}\right)^{s}} \int_{S(a)}\left(1-|z|^{2}\right)^{s}\left|g^{\prime}(z) f_{a}(z)\right|^{2} d A(z) \\
= & \frac{1}{\left(1-|a|^{2}\right)^{s}} \int_{S(a)}\left(1-|z|^{2}\right)^{s}\left|\left(T_{g}\left(f_{a}\right)\right)^{\prime}(z)\right|^{2} d A(z) .
\end{aligned}
$$

The fact that $T_{g}$ is a bounded operator from $B^{p}$ into $Q_{s}$, implies that the measures $\left(1-|z|^{2}\right)^{s}\left|\left(T_{g}\left(f_{a}\right)\right)^{\prime}(z)\right|^{2} d A(z)$ are $s$-Carleson measures with constants controlled by $\left\|T_{g}\right\|^{2}$. Then it follows that the measure $\left(1-|z|^{2}\right)^{s}\left|g^{\prime}(z)\right|^{2} d A(z)$ is a $2 / p^{\prime}$ logarithmic $s$-Carleson measure and, hence, $g \in Q_{s, \log , 1 / p^{\prime}}$.

Proof of Theorem 2.4 (iii) and (iv). In view of (ii) we only have to prove that if $g \in Q_{s, \log , 1 / p^{\prime}}$ then $T_{g}$ maps $B^{p}$ into $Q_{s}$.

Hence, take $g \in Q_{s, \log , 1 / p^{\prime}}$ and set

$$
K(g)=\sup _{a \in \mathbb{D}}\left(\log \frac{2}{1-|a|}\right)^{2 / p^{\prime}} \int_{\mathbb{D}}\left|g^{\prime}(z)\right|^{2}\left(1-\left|\varphi_{a}(z)\right|^{2}\right)^{s} d A(z),
$$

and take $f \in B^{p}$. Set $F=T_{g}(f)$, we have to prove that $F \in Q_{s}$ or, equivalently, that the measure $\mu_{F}$ defined by

$$
d \mu_{F}(z)=\left(1-|z|^{2}\right)^{s}\left|F^{\prime}(z)\right|^{2} d A(z)
$$

is an $s$-Carleson measure. Let $a \in \mathbb{D}$. Using the well known fact that

$$
1-|a|^{2} \asymp|1-\bar{a} z|, \quad z \in S(a),
$$

we obtain

$$
\begin{aligned}
& \frac{1}{\left(1-|a|^{2}\right)^{s}} \int_{S(a)}\left|F^{\prime}(z)\right|^{2}\left(1-|z|^{2}\right)^{s} d A(z) \asymp \int_{S(a)}\left|F^{\prime}(z)\right|^{2} \frac{\left(1-|z|^{2}\right)^{s}\left(1-|a|^{2}\right)^{s}}{|1-\bar{a} z|^{2 s}} d A(z) \\
& =\int_{S(a)}|f(z)|^{2}\left|g^{\prime}(z)\right|^{2}\left(1-\left|\varphi_{a}(z)\right|^{2}\right)^{s} d A(z) \\
& \leq 2 \int_{\mathbb{D}}|f(a)|^{2}\left|g^{\prime}(z)\right|^{2}\left(1-\left|\varphi_{a}(z)\right|^{2}\right)^{s} d A(z) \\
& \quad+2 \int_{\mathbb{D}}|f(z)-f(a)|^{2}\left|g^{\prime}(z)\right|^{2}\left(1-\left|\varphi_{a}(z)\right|^{2}\right)^{s} d A(z) \\
& =2 T_{1}(a)+2 T_{2}(a) .
\end{aligned}
$$

Using the fact that

$$
|f(a)-f(0)| \lesssim\|f\|_{B^{p}}\left(\log \frac{2}{1-|a|^{2}}\right)^{1 / p^{\prime}},
$$


we obtain

$$
T_{1}(a) \lesssim\|f\|_{B^{p}}^{2}\left(\log \frac{2}{1-|a|^{2}}\right)^{2 / p^{\prime}} \int_{\mathbb{D}}\left|g^{\prime}(z)\right|^{2}\left(1-\left|\varphi_{a}(z)\right|^{2}\right)^{s} d A(z) \lesssim K(g)\|f\|_{B^{p}}^{2}
$$

To estimate $T_{2}(a)$ we shall treat separately the cases $s=1$ and $0<s<1$.

Let us start with the case $s=1$. Then

$$
T_{2}(a)=\int_{\mathbb{D}}|f(z)-f(a)|^{2}\left|g^{\prime}(z)\right|^{2}\left(1-\left|\varphi_{a}(z)\right|^{2}\right) d A(z) .
$$

Making the change of variable $w=\varphi(z)$ in the last integral, we obtain

$$
T_{2}(a)=\int_{\mathbb{D}}\left|\left(f \circ \varphi_{a}\right)(w)-f(a)\right|^{2}\left|\left(g \circ \varphi_{a}\right)^{\prime}(w)\right|^{2}\left(1-|w|^{2}\right) d A(w) .
$$

Since $Q_{1, \log , 1 / p^{\prime}} \subset Q_{1}=B M O A, g \in B M O A$ and then it follows that, for all $a \in \mathbb{D}, g \circ \varphi_{a} \in B M O A$ and $\rho_{*}\left(g \circ \varphi_{a}\right)=\rho_{*}(g)$. This gives that all the measures $\left(1-|w|^{2}\right)\left|\left(g \circ \varphi_{a}\right)^{\prime}(w)\right|^{2} d A(w)(a \in \mathbb{D})$ are Carleson measures with constants controlled by $\|g\|_{B M O A}^{2}$. Then, using the Carleson embedding theorem for $H^{2}$ and the fact that $B^{p}$ is continuously embedded in $B M O A$, it follows that

$$
T_{2}(a) \lesssim\|g\|_{B M O A}^{2}\left\|f \circ \varphi_{a}-f(a)\right\|_{H^{2}}^{2} \lesssim\|g\|_{B M O A}^{2}\|f\|_{B M O A}^{2} \lesssim\|g\|_{B M O A}^{2}\|f\|_{B^{p}}^{2} .
$$

Putting together this, (2.5), and (2.7), we see that the measure $\mu_{F}$ is a Carleson measure. This finishes the proof of part (iii).

To finish the proof of part (iv) we proceed to estimate $T_{2}(a)$ assuming that $2<p<\infty, 0<s<1$, and $1-\frac{2}{p}<s$. Notice that

$$
T_{2}(a)=\left(1-|a|^{2}\right)^{s} \int_{\mathbb{D}}\left|\frac{f(z)-f(a)}{(1-\bar{a} z)^{s}}\right|^{2}\left|g^{\prime}(z)\right|^{2}\left(1-|z|^{2}\right)^{s} d A(z) .
$$

Since $0<s<1,2 / p^{\prime}>1$, and the measure $\left(1-|z|^{2}\right)^{s}\left|g^{\prime}(z)\right|^{2} d A(z)$ is a $2 / p^{\prime}$ logarithmic $s$-Carleson measure, using Theorem B (v), it follows that

$$
T_{2}(a) \lesssim\left(1-|a|^{2}\right)^{s}\left(|f(a)-f(0)|^{2}+\int_{\mathbb{D}}\left|\left(\frac{f(z)-f(a)}{(1-\bar{a} z)^{s}}\right)^{\prime}\right|^{2}\left(1-|z|^{2}\right)^{s} d A(z)\right) \text {. }
$$

The growth estimate (2.6) and simple computations yield

$$
\begin{aligned}
T_{2}(a) & \lesssim\|f\|_{B^{p}}^{2}\left(1-|a|^{2}\right)^{s}\left(\log \frac{2}{1-|a|^{2}}\right)^{2 / p^{\prime}}+\int_{\mathbb{D}}\left|f^{\prime}(z)\right|^{2}\left(1-\left|\varphi_{a}(z)\right|^{2}\right)^{s} d A(z) \\
& +\int_{\mathbb{D}} \frac{|f(z)-f(a)|^{2}}{|1-\bar{a} z|^{2}}\left(1-\left|\varphi_{a}(z)\right|^{2}\right)^{s} d A(z) \\
& \lesssim\|f\|_{B^{p}}^{2}+\int_{\mathbb{D}}\left|f^{\prime}(z)\right|^{2}\left(1-\left|\varphi_{a}(z)\right|^{2}\right)^{s} d A(z)+\int_{\mathbb{D}} \frac{|f(z)-f(a)|^{2}}{|1-\bar{a} z|^{2}}\left(1-\left|\varphi_{a}(z)\right|^{2}\right)^{s} d A(z) .
\end{aligned}
$$

By Theorem B (iv), our assumptions on $s$ and $p$ imply that $B^{p}$ is continuously embedded in $Q_{s}$. Hence, $f \in Q_{s}$. This implies that

$$
\int_{\mathbb{D}}\left|f^{\prime}(z)\right|^{2}\left(1-\left|\varphi_{a}(z)\right|^{2}\right)^{s} d A(z) \leq\|f\|_{Q_{s}}^{2} \lesssim\|f\|_{B^{p}}^{2}
$$


and that

$$
\int_{\mathbb{D}} \frac{|f(z)-f(a)|^{2}}{|1-\bar{a} z|^{2}}\left(1-\left|\varphi_{a}(z)\right|^{2}\right)^{s} d A(z) \lesssim\|f\|_{Q_{s}}^{2} \lesssim\|f\|_{B^{p}}^{2},
$$

by a result proved by Pau and Peláez in [41, pp. 551-552]. Consequently, we have proved that $T_{2}(a) \lesssim\|f\|_{B^{p}}^{2}$. This, together with (2.5) and (2.7), shows that $\mu_{F}$ is an $s$-Carleson measure as desired. Thus the proof is also finished in this case.

The case when $1<p \leq 2$ and $0<s<1$ remains open. This is so because if we set $\alpha=2 / p^{\prime}$, then $\alpha \leq 1$ and, hence, $\alpha$ is not in the conditions of Theorem B (v). We can prove the following result.

Theorem 2.5. Suppose that $1<p \leq 2$ and $0<s<1$, and let $g \in \mathcal{H o l}(\mathbb{D})$. The following statements hold.

(i) If $T_{g}$ maps $B^{p}$ into $Q_{s}$ then $g \in Q_{s, \log , 1 / p^{\prime}}$.

(ii) If $\alpha>1 / 2$ and $g \in Q_{s, \log , \alpha}$ then $T_{g}$ maps $B^{p}$ into $Q_{s}$.

Proof. (i) follows from part (ii) of Theorem 2.4. Set

Let us turn to prove (ii). Suppose that $0<s<1, \alpha>1 / 2$, and $g \in Q_{s, \log , \alpha}$.

$$
K(g)=\sup _{a \in \mathbb{D}}\left(\log \frac{2}{1-|a|}\right)^{2 \alpha} \int_{\mathbb{D}}\left|g^{\prime}(z)\right|^{2}\left(1-\left|\varphi_{a}(z)\right|^{2}\right)^{s} d A(z),
$$

and take $f \in B^{p}$. Set $F=T_{g}(f)$, we have to prove the $F \in Q_{s}$ or, equivalently, that the measure $\mu_{F}$ defined by

$$
d \mu_{F}(z)=\left(1-|z|^{2}\right)^{s}\left|F^{\prime}(z)\right|^{2} d A(z)
$$

is an $s$-Carleson measure. Now we argue as in the proof of Theorem 2.4(iv). For $a \in \mathbb{D}$, we obtain

$$
\frac{1}{\left(1-|a|^{2}\right)^{s}} \int_{S(a)}\left|F^{\prime}(z)\right|^{2}\left(1-|z|^{2}\right)^{s} d A(z) \lesssim 2 T_{1}(a)+2 T_{2}(a),
$$

where $T_{1}(a)$ and $T_{2}(a)$ are defined as in the proof of Theorem 2.4. Using (2.6) and the fact that $\frac{1}{p^{\prime}} \leq \frac{1}{2}<\alpha$, we obtain

$$
|f(a)-f(0)| \lesssim\|f\|_{B^{p}}\left(\log \frac{2}{1-|a|^{2}}\right)^{\alpha} .
$$

This yields

$$
T_{1}(a) \lesssim\|f\|_{B^{p}}^{2}\left(\log \frac{2}{1-|a|^{2}}\right)^{2 \alpha} \int_{\mathbb{D}}\left|g^{\prime}(z)\right|^{2}\left(1-\left|\varphi_{a}(z)\right|^{2}\right)^{s} d A(z) \lesssim K(g)\|f\|_{B^{p}}^{2} .
$$

To estimate $T_{2}(a)$, observe that the measure $\left(1-|z|^{2}\right)^{s}\left|g^{\prime}(z)\right|^{2} d A(z)$ is a $2 \alpha$ logarithmic $s$-Carleson measure. Since $2 \alpha>1$, using Lemma 1 of [41], this implies that the measure $\left(1-|z|^{2}\right)^{s}\left|g^{\prime}(z)\right|^{2} d A(z)$ is a Carleson measure for $\mathcal{D}_{s}$. Then, arguing as in the proof of Theorem 2.4 (iv), we obtain $T_{2}(a) \lesssim\|f\|_{B^{p}}^{2}$. This, together with (2.9) and (2.8), implies that the measure $\mu_{F}$ is an $s$-Carleson measure. 
Regarding the operators $I_{g}$ and $M_{g}$ we have the following results.

Theorem 2.6. Let $g \in \mathcal{H}$ ol $(\mathbb{D})$, then:

(1) If $1<p \leq 2$ and $0<s \leq 1$ then:

(1a) $I_{g}$ maps $B^{p}$ into $Q_{s}$ if and only if $g \in H^{\infty}$.

(1b) If $M_{g}$ maps $B^{p}$ into $Q_{s}$ then $g \in Q_{s, \log , 1 / p^{\prime}} \cap H^{\infty}$.

(1c) If $g \in Q_{s, \log , \alpha} \cap H^{\infty}$ for some $\alpha>1 / 2$ then $M_{g}$ maps $B^{p}$ into $Q_{s}$.

(2) If $2<p<\infty$ and $1-\frac{2}{p}<s \leq 1$ then:

(2a) $I_{g}$ maps $B^{p}$ into $Q_{s}$ if and only if $g \in H^{\infty}$.

(2b) $M_{g}$ maps $B^{p}$ into $Q_{s}$ if and only if $g \in Q_{s, \log , 1 / p^{\prime}} \cap H^{\infty}$.

(3) If $2<p<\infty$ and $0<s \leq 1-\frac{2}{p}$ then:

(3a) $I_{g}$ maps $B^{p}$ into $Q_{s}$ if and only if $g \equiv 0$.

(3b) $M_{g}$ maps $B^{p}$ into $Q_{s}$ if and only if $g \equiv 0$.

Proof of Parts (1) and (2) of Theorem 2.6. Using Proposition 1.1 it follows that if either $I_{g}$ or $M_{g}$ maps $B^{p}$ into $Q_{s}$ for any pair $(s, p)$ with $0<s<\infty$ and $1<p<\infty$ then $g \in H^{\infty}$.

Suppose now that $s$ and $p$ are in the conditions of (1) or (2) and that $g \in H^{\infty}$. Take $f \in B^{p}$. We have to prove $I_{g}(f) \in Q_{s}$ or, equivalently, that the measure

$$
\left(1-|z|^{2}\right)^{s}\left|f^{\prime}(z)\right|^{2}|g(z)|^{2} d A(z) \text { is an } s \text {-Carleson measure. }
$$

Using (1.1) and (1.2), we see that $B^{p} \subset Q_{s}$. Hence $f \in Q_{s}$ which is the same as saying that $\left(1-|z|^{2}\right)^{s}\left|f^{\prime}(z)\right|^{2} d A(z)$ is an $s$-Carleson measure. This and the fact that $g \in H^{\infty}$ trivially yield (2.10). Thus (1a) and (2a) are proved. Then (1b), (1c), and (2b) follow using Proposition 1.1, the fact that if two of the operators $T_{g}, I_{g}, M_{g}$ map $B^{p}$ into $Q_{s}$ so does the third one, Theorem 2.4, and Theorem 2.5.

In order to prove Theorem 2.6 (3), for $2<p<\infty$ we shall consider the function $F_{p}$ defined by

$$
F_{p}(z)=\sum_{k=1}^{\infty} \frac{1}{k^{1 / 2} 2^{k / p}} z^{2^{k}}, \quad z \in \mathbb{D} .
$$

Using [10, Corollary 7] or [14, Theorem 6], we see that $F_{p} \in B^{p}$ and $F_{p} \notin Q_{1-\frac{2}{p}}$. Hence

$$
F_{p} \in B^{p} \backslash Q_{s}, \quad 0<s \leq 1-\frac{2}{p}, \quad 2<p<\infty .
$$

Let us estimate the integral means $M_{2}\left(r, F_{p}^{\prime}\right)$. We have

$$
z F_{p}^{\prime}(z)=\sum_{k=1}^{\infty} 2^{k / p^{\prime}} k^{-1 / 2} z^{2^{k}}, \quad z \in \mathbb{D}
$$

and, hence,

$$
M_{2}\left(r, F_{p}^{\prime}\right)^{2} \gtrsim \sum_{k=1}^{\infty} 2^{2 k / p^{\prime}} k^{-1} r^{2^{k+1}}, \quad 0<r<1 .
$$


Set $r_{n}=1-2^{-n}(n=1,2, \ldots)$. Then

$$
\begin{aligned}
& M_{2}\left(r_{n}, F_{p}^{\prime}\right)^{2} \gtrsim \sum_{k=1}^{\infty} 2^{2 k / p^{\prime}} k^{-1} r_{n}^{2^{k+1}} \\
& \gtrsim 2^{2 n / p^{\prime}} n^{-1} r_{n}^{2^{n+1}} \gtrsim 2^{2 n / p^{\prime}} n^{-1} \asymp \frac{1}{\left(1-r_{n}\right)^{2 / p^{\prime}} \log \frac{2}{1-r_{n}}}, \quad n=1,2, \ldots
\end{aligned}
$$

This readily yields

$$
M_{2}\left(r, F_{p}^{\prime}\right)^{2} \gtrsim \frac{1}{(1-r)^{2 / p^{\prime}} \log \frac{2}{1-r}}, \quad 0<r<1 .
$$

Proof of part (3) of Theorem 2.6. Suppose that $2<p<\infty$ and $0<s \leq 1-\frac{2}{p}$ and $g \in \mathcal{H o l}(\mathbb{D})$ is not identically zero.

Suppose first that either $I_{g}$ or $M_{g}$ maps $B^{p}$ into $Q_{s}$. We know that then $g \in H^{\infty}$ and then, by Fatou's theorem and the Riesz uniqueness theorem, we know that $g$ has a finite non-tangential limit $g\left(e^{i \theta}\right)$ for almost every $\theta \in[0,2 \pi]$ and that $g\left(e^{i \theta}\right) \neq 0$ for almost every $\theta$. Then it follows that there exist $C>0, r_{0} \in(0,1)$, and a measurable set $E \subset[0,2 \pi]$ whose Lebesgue measure $|E|$ is positive such that

$$
\left|g\left(r e^{i \theta}\right)\right| \geq C, \quad \theta \in E, \quad r_{0}<r<1 .
$$

Since $F_{p}$ is given by a power series with Hadamard gaps, Lemma 6. 5 in [60, Vol. 1 , p. 203] implies that

$$
\int_{E}\left|F_{p}^{\prime}\left(r e^{i \theta}\right)\right|^{2} d \theta \asymp M_{2}\left(r, F_{p}^{\prime}\right)^{2}, \quad 0<r<1 .
$$

Using the fact that $s \leq 1-\frac{2}{p},(2.14),(2.15)$, and (2.13), we obtain

$$
\begin{aligned}
& \int_{0}^{1}(1-r)^{s} M_{2}\left(r, F_{p}^{\prime} g\right)^{2} d r \geq \int_{r_{0}}^{1}(1-r)^{1-\frac{2}{p}} M_{2}\left(r, F_{p}^{\prime} g\right)^{2} d r \\
\gtrsim & \int_{r_{0}}^{1}(1-r)^{1-\frac{2}{p}} \int_{E}\left|F_{p}^{\prime}\left(r e^{i \theta}\right)\right|^{2}\left|g\left(r e^{i \theta}\right)\right|^{2} d \theta d r \gtrsim \int_{r_{0}}^{1}(1-r)^{1-\frac{2}{p}} \int_{E}\left|F_{p}^{\prime}\left(r e^{i \theta}\right)\right|^{2} d \theta d r \\
\gtrsim & \int_{r_{0}}^{1}(1-r)^{1-\frac{2}{p}} M_{2}\left(r, F_{p}^{\prime}\right)^{2} d r \gtrsim \int_{r_{0}}^{1} \frac{d r}{(1-r) \log \frac{2}{1-r}}=\infty .
\end{aligned}
$$

If we assume that $I_{g}$ maps $B^{p}$ into $Q_{s}$ then $I_{g}\left(F_{p}\right) \in Q_{s}$ and then, using [11, Proposition 3.1], it follows that

$$
\int_{0}^{1}(1-r)^{s} M_{2}\left(r, F_{p}^{\prime} g\right)^{2} d r<\infty .
$$

This is in contradiction with (2.16).

Assume now that $M_{g}$ maps $B^{p}$ into $Q_{s}$. Since 1 and $F_{p}$ belong to $B^{p}$, we have that $g$ and $F_{p} g$ belong to $Q_{s}$ and then, by [11, Proposition 3. 1],

$$
\int_{0}^{1}(1-r)^{s} M_{2}\left(r, g^{\prime}\right)^{2} d r<\infty
$$


and

$$
\int_{0}^{1}(1-r)^{s} M_{2}\left(r,\left(F_{p} g\right)^{\prime}\right)^{2} d r<\infty .
$$

Notice that $F_{p} \in H^{\infty}$ and then

$$
M_{2}\left(r, F_{p} g^{\prime}\right) \lesssim M_{2}\left(r, g^{\prime}\right), \quad 0<r<1 .
$$

This and (2.17) imply that

$$
\int_{0}^{1}(1-r)^{s} M_{2}\left(r, F_{p}^{\prime} g\right)^{2} d r<\infty .
$$

We have arrived to a contradiction because it is clear that (2.16) and (2.19) cannot be simultaneously true.

In the other direction we have the following result.

Theorem 2.7. Suppose that $0<s<\infty$ and $1 \leq p<\infty$ and let $g \in \mathcal{H o l}(\mathbb{D})$. Then the following conditions are equivalent

(i) $M_{g}$ maps $Q_{s}$ into $B^{p}$.

(ii) $g \equiv 0$.

Proof. Suppose that $g \not \equiv 0$. Choose an increasing sequence $\left\{r_{n}\right\}_{n=1}^{\infty} \subset(0,1)$ with $\lim \left\{r_{n}\right\}=1$ and a sequence $\left\{\theta_{n}\right\}_{n=1}^{\infty} \subset[0,2 \pi]$ such that

$$
\left|g\left(r_{n} e^{i \theta_{n}}\right)\right|=M_{\infty}\left(r_{n}, g\right), \quad n=1,2, \ldots
$$

For each $n$ set

$$
f_{n}(z)=\log \frac{1}{1-e^{-i \theta_{n} z}}, \quad z \in \mathbb{D} .
$$

Notice that $M\left(r_{1}, g\right)>0$ and that the sequence $\left\{M\left(r_{n}, g\right)\right\}$ is increasing. Set

$$
f_{n}(z)=\log \frac{1}{1-e^{-i \theta_{n} z}}, \quad z \in \mathbb{D}, \quad n=1,2, \ldots
$$

We have that $f_{n} \in Q_{s}$ for all $n$ and

$$
\left\|f_{n}\right\|_{Q_{s}} \asymp 1 \text {. }
$$

Assume that $M_{g}$ maps $Q_{s}$ into $B^{p}$. Then, by the closed graph theorem, $M_{g}$ is bounded operator from $Q_{s}$ into $B^{p}$. Hence the sequence $\left\{g f_{n}\right\}_{n=1}^{\infty}$ is a bounded sequence on $B^{p}$, that is,

$$
\left\|g f_{n}\right\|_{B^{p}} \lesssim 1
$$

Then it follows that

$$
\begin{aligned}
M\left(r_{1}, g\right) \log \frac{1}{1-r_{n}} & \leq M\left(r_{n}, g\right) \log \frac{1}{1-r_{n}}=\left|g\left(r_{n} e^{i \theta_{n}}\right) f_{n}\left(r_{n} e^{i \theta_{n}}\right)\right| \\
& \lesssim\left\|g f_{n}\right\|_{B^{p}}\left(\log \frac{1}{1-r_{n}}\right)^{1 / p^{\prime}} \lesssim\left(\log \frac{1}{1-r_{n}}\right)^{1 / p^{\prime}} .
\end{aligned}
$$

This is a contradiction. 


\section{Multipliers And integration operators Between $Q_{s}$ SPACES}

As we mentioned above the space of multipliers $M(\mathcal{B})=M\left(Q_{s}\right)(s>1)$ was characterized by Brown and Shields in [15]. Ortega and Fàbrega [40] characterized the space $M(B M O A)=M\left(Q_{1}\right)$. Pau and Peláez [41] and Xiao [54] characterized the spaces $M\left(Q_{s}\right)(0<s<1)$ closing a conjecture formulated in [51]. Indeed, Theorem 1 of [41] and Theorem 1.2 of [54] assert the following.

Theorem C. Suppose that $0<s \leq 1$ and let $g$ be an analytic function in the unit disc $\mathbb{D}$. Then:

(i) $T_{g}$ maps $Q_{s}$ into itself if and only if $g \in Q_{s, \log , 1}$.

(ii) $I_{g}$ maps $Q_{s}$ into itself if and only if $g \in H^{\infty}$.

(ii) $M_{g}$ maps $Q_{s}$ into itself if and only if $g \in Q_{s, \log , 1} \cap H^{\infty}$.

We shall prove the following results.

Theorem 3.1. Suppose that $0<s_{1} \leq s_{2} \leq 1$ and let $g \in \mathcal{H}$ ol $(\mathbb{D})$. Then:

(i) $T_{g}$ maps $Q_{s_{1}}$ into $Q_{s_{2}}$ if and only if $g \in Q_{s_{2}, \log , 1}$.

(ii) $I_{g}$ maps $Q_{s_{1}}$ into $Q_{s_{2}}$ if and only if $g \in H^{\infty}$.

(iii) $M_{g}$ maps $Q_{s_{1}}$ into $Q_{s_{2}}$ if and only if $g \in Q_{s_{2}, \log , 1} \cap H^{\infty}$.

Theorem 3.2. Suppose that $0<s_{1}<s_{2} \leq 1$ and let $g \in \mathcal{H o l}(\mathbb{D})$. Then the following conditions are equivalent:

(i) $I_{g}$ maps $Q_{s_{2}}$ into $Q_{s_{1}}$.

(ii) $M_{g}$ maps $Q_{s_{2}}$ into $Q_{s_{1}}$.

(iii) $g \equiv 0$.

Proof of Theorem 3.1. For $a \in \mathbb{D}$ we set

$$
h_{a}(z)=\log \frac{2}{1-\bar{a} z}, \quad z \in \mathbb{D} .
$$

Then $h_{a} \in Q_{s_{1}}$ for all $a \in \mathbb{D}$ and

$$
\left\|h_{a}\right\|_{Q_{s_{1}}} \asymp 1 \text {. }
$$

- If $T_{g}$ maps $Q_{s_{1}}$ into $Q_{s_{2}}$ then $T_{g}$ is a bounded operator from $Q_{s_{1}}$ into $Q_{s_{2}}$. Using this and (3.1), it follows that for all $a \in \mathbb{D}$ the measure (1 $\left.|z|^{2}\right)^{s_{2}}\left|g^{\prime}(z)\right|^{2}\left|h_{a}(z)\right|^{2} d A(z)$ is an $s_{2}$-Carleson measure and that

$$
\int_{S(a)}\left(1-|z|^{2}\right)^{s_{2}}\left|g^{\prime}(z)\right|^{2}\left|h_{a}(z)\right|^{2} d A(z) \lesssim\left(1-|a|^{2}\right)^{s_{2}}, \quad a \in \mathbb{D} .
$$

Since

(3.2) implies that

$$
\left|h_{a}(z)\right| \asymp \log \frac{2}{1-|a|^{2}}, \quad z \in S(a)
$$

$$
\left(\log \frac{2}{1-|a|^{2}}\right)^{2} \int_{S(a)}\left(1-|z|^{2}\right)^{s_{2}}\left|g^{\prime}(z)\right|^{2} d A(z) \lesssim\left(1-|a|^{2}\right)^{s_{2}} .
$$


This is the same as saying that the measure $\left(1-|z|^{2}\right)^{s_{2}}\left|g^{\prime}(z)\right|^{2} d A(z)$ is a 2logarithmic $s_{2}$-Carleson measure or, equivalently, that $g \in Q_{s_{2}, \log , 1}$.

If $g \in Q_{s_{2}, \log , 1}$ then, by Theorem C, $T_{g}$ maps $Q_{s_{2}}$ into itself. Since $Q_{s_{1}} \subset Q_{s_{2}}$, it follows trivially that $T_{g}$ maps $Q_{s_{1}}$ into $Q_{s_{2}}$. Hence (i) is proved

- Proposition 1.1 shows that if $I_{g}$ maps $Q_{s_{1}}$ into $Q_{s_{2}}$ then $g \in H^{\infty}$.

Conversely, suppose that $g \in H^{\infty}$. In order to prove that $I_{g}$ maps $Q_{s_{1}}$ into $Q_{s_{2}}$, we have to prove that for any $f \in Q_{s_{1}}$ the measure $\left(1-|z|^{2}\right)^{s_{2}}|g(z)|^{2}\left|f^{\prime}(z)\right|^{2} d A(z)$ is an $s_{2}$-Carleson measure. So, take $f \in Q_{s_{1}}$. Then $\left(1-|z|^{2}\right)^{s_{1}}\left|f^{\prime}(z)\right|^{2} d A(z)$ is an $s_{1}$-Carleson measure. Then it follows that

$$
\begin{aligned}
& \int_{S(a)}\left(1-|z|^{2}\right)^{s_{2}}|g(z)|^{2}\left|f^{\prime}(z)\right|^{2} d A(z) \\
\leq & \|g\|_{H^{\infty}}^{2}\left(1-|a|^{2}\right)^{s_{2}-s_{1}} \int_{S(a)}\left(1-|z|^{2}\right)^{s_{1}}\left|f^{\prime}(z)\right|^{2} d A(z) \\
\lesssim & \left(1-|a|^{2}\right)^{s_{2}} .
\end{aligned}
$$

This shows that $\left(1-|z|^{2}\right)^{s_{2}}|g(z)|^{2}\left|f^{\prime}(z)\right|^{2} d A(z)$ is an $s_{2}$-Carleson measure as desired, finishing the proof of (ii).

- If $M_{g}$ maps $Q_{s_{1}}$ into $Q_{s_{2}}$ then, Proposition 1.1, $g \in H^{\infty}$. Then (i) implies that $I_{g}$ maps $Q_{s_{1}}$ into $Q_{s_{2}}$. Since $M_{g}(f)=I_{g}(f)+T_{g}(f)+f(0) g(0)$, it follows that $T_{g}$ maps $Q_{s_{1}}$ into $Q_{s_{2}}$. Then (i) yields $g \in Q_{s_{2}, \log , 1}$. Then we have that $g \in Q_{s_{2}, \log , 1} \cap H^{\infty}$.

Conversely, if $g \in Q_{s_{2}, \log , 1} \cap H^{\infty}$ then (i) and (ii) immediately give that both $T_{g}$ and $I_{g}$ map $Q_{s_{1}}$ into $Q_{s_{2}}$ and then so does $M_{g}$.

Some results from [11] will be used to prove Theorem 3.2. As we have already noticed if $0<s \leq 1$ and $f \in Q_{s}$ then $\int_{0}^{1}(1-r)^{s} M_{2}\left(r, f^{\prime}\right)^{2} d r<\infty$. Using ideas from [27], Aulaskari, Girela and Wulan [11, Theorem 3. 1] proved that this result is sharp in a very strong sense.

Theorem D. Suppose that $0<s \leq 1$ and let $\varphi$ be a positive increasing function defined in $(0,1)$ such that

$$
\int_{0}^{1}(1-r)^{s} \varphi(r)^{2} d r<\infty .
$$

Then there exists a function $f \in Q_{s}$ given by a power series with Hadamard gaps such that $M_{2}\left(r, f^{\prime}\right) \geq \varphi(r)$ for all $r \in(0,1)$.

Proof of Theorem 3.2. Suppose that $g \not \equiv 0$ and that either $I_{g}$ or $M_{g}$ maps $Q_{s_{2}}$ into $Q_{s_{1}}$. By Proposition 1.1, $g \in H^{\infty}$ and then it follows that there exist $C>0$, $r_{0} \in(0,1)$, and a measurable set $E \subset[0,2 \pi]$ whose Lebesgue measure $|E|$ is positive such that

$$
\left|g\left(r e^{i \theta}\right)\right| \geq C, \quad \theta \in E, \quad r_{0}<r<1 .
$$

- Suppose that $I_{g}$ maps $Q_{s_{2}}$ into $Q_{s_{1}}$. Then we use Theorem D to pick a function $F \in Q_{s_{2}}$ given by a power series with Hadamard gaps so that

$$
M_{2}\left(r, F^{\prime}\right) \geq \frac{1}{(1-r)^{\left(1+s_{1}\right) / 2}}, \quad 0<r<1 .
$$


Since $I_{g}(F) \in Q_{s_{1}}$,

$$
\int_{0}^{1}(1-r)^{s_{1}} M_{2}\left(r, F^{\prime} g\right)^{2} d r<\infty .
$$

However, using Lemma 6.5 in [60, Vol. 1, p. 203] and (3.3), it follows that

$$
\begin{aligned}
\int_{0}^{1}(1-r)^{s_{1}} M_{2}\left(r, F^{\prime} g\right)^{2} d r & \gtrsim \int_{r_{0}}^{1}(1-r)^{s_{1}} \int_{E}\left|F^{\prime}\left(r e^{i \theta}\right)\right|^{2}\left|g\left(r e^{i \theta}\right)\right|^{2} d \theta d r \\
& \gtrsim \int_{r_{0}}^{1}(1-r)^{s_{1}} \int_{E}\left|F^{\prime}\left(r e^{i \theta}\right)\right|^{2} d \theta d r \\
& \asymp \int_{r_{0}}^{1}(1-r)^{s_{1}} M_{2}\left(r, F^{\prime}\right)^{2} d r \\
& \gtrsim \int_{r_{0}}^{1}(1-r)^{-1} d r \\
& =\infty .
\end{aligned}
$$

This is in contradiction with (3.4).

- Suppose now that $M_{g}$ maps $Q_{s_{2}}$ into $Q_{s_{1}}$. Take $\varepsilon>0$ with $s_{2}-s_{1}-\varepsilon>0$ and use Theorem $\mathrm{D}$ to pick a function $H \in Q_{s_{2}}$ given by a power series with Hadamard gaps so that

$$
M_{2}\left(r, H^{\prime}\right) \geq \frac{1}{(1-r)^{\left(1+s_{1}+\varepsilon\right) / 2}}, \quad 0<r<1 .
$$

Since $g H \in Q_{s_{1}}$ we have that

$$
\int_{0}^{1}(1-r)^{s_{1}} M_{2}\left(r, g^{\prime} H+g H^{\prime}\right)^{2} d r<\infty .
$$

Using Lemma 6.5 in [60, Vol. 1, p. 203] and (3.5), we obtain as above that

$$
\begin{aligned}
\int_{0}^{1}(1-r)^{s_{1}+\varepsilon} M_{2}\left(r, H^{\prime} g\right)^{2} d r & \gtrsim \int_{r_{0}}^{1}(1-r)^{s_{1}+\varepsilon} \int_{E}\left|H^{\prime}\left(r e^{i \theta}\right)\right|^{2} d \theta d r \\
& \gtrsim \int_{r_{0}}^{1}(1-r)^{s_{1}+\varepsilon} M_{2}\left(r, H^{\prime}\right)^{2} d r \\
& \gtrsim \int_{r_{0}}^{1} \frac{d r}{1-r} \\
& =\infty .
\end{aligned}
$$

Notice that $g \in Q_{s_{1}}$. Using this and the fact that

it follows that

$$
|H(z)| \lesssim \log \frac{2}{1-|z|}, \quad z \in \mathbb{D}
$$

$$
\begin{aligned}
& \int_{0}^{1}(1-r)^{s_{1}+\varepsilon} M_{2}\left(r, H g^{\prime}\right)^{2} d r \lesssim \int_{0}^{1}(1-r)^{s_{1}+\varepsilon}\left(\log \frac{2}{1-r}\right)^{2} M_{2}\left(r, g^{\prime}\right)^{2} d r \\
& \lesssim \int_{0}^{1}(1-r)^{s_{1}+\frac{\varepsilon}{2}} M_{2}\left(r, g^{\prime}\right) d r<\infty
\end{aligned}
$$


We have arrived to a contradiction because (3.6), (3.7), and (3.8) cannot hold simultaneously.

Remark 3.3. The implication (ii) $\Rightarrow$ (iii) in Theorem 3.2 was obtained by Pau and Peláez [42, Corollary 4] using the fact that there exists a function $f \in Q_{s_{2}}$, $f \not \equiv 0$, whose sequence of zeros is not a $Q_{s_{1}}$-zero set.

This idea gives also the following:

$$
M\left(\mathcal{B}, Q_{s}\right)=\{0\}, \quad 0<s \leq 1 .
$$

Indeed, it is well known that there exists a function $f \in \mathcal{B}, f \not \equiv 0$, whose sequence of zeros does not satisfy the Blaschke condition [7,31]. If $g \not \equiv 0$ were a multiplier from $\mathcal{B}$ into $Q_{s}$ for some $s \leq 1$ then the sequence of zeros of $f g$ would satisfy the Blaschke condition. But this is not true because all the zeros of $f$ are zeros of $g f$.

\section{Some FURTHER RESUlts}

The inner-outer factorization of functions in the Hardy spaces plays an outstanding role in lots of questions. In many cases the outer factor $O_{f}$ of $f$ inherits properties of $f$. Working in this setting the following concepts arise as natural and quite interesting.

A subspace $X$ of $H^{1}$ is said to have the $f$-property (also called the property of division by inner functions) if $h / I \in X$ whenever $h \in X$ and $I$ is an inner function with $h / I \in H^{1}$.

Given $v \in L^{\infty}(\partial \mathbb{D})$, the Toeplitz operator $T_{v}$ associated with the symbol $v$ is defined by

$$
T_{v} f(z)=P(v f)(z)=\frac{1}{2 \pi i} \int_{\partial \mathbb{D}} \frac{v(\xi) f(\xi)}{\xi-z} d \xi, \quad f \in H^{1}, \quad z \in \mathbb{D} .
$$

Here, $P$ is the Szegö projection.

A subspace $X$ of $H^{1}$ is said to have the $K$-property if $T_{\bar{\psi}}(X) \subset X$ for any $\psi \in H^{\infty}$.

These notions were introduced by Havin in [34]. It was also pointed out in [34] that the $K$-property implies the $f$-property: indeed, if $h \in H^{1}, I$ is inner and $h / I \in H^{1}$ then $h / I=T_{\bar{I}} h$.

In addition to the Hardy spaces $H^{p}(1<p<\infty)$ many other spaces such as the Dirichlet space [34, 38], several spaces of Dirichlet type including all the Besov spaces $B^{p}(1<p<\infty)[20,21,22,39]$, the spaces $B M O A$ and $V M O A$ [35], and the $Q_{s}$ spaces $(0<s<1)[23]$ have the $K$-property. The Hardy space $H^{1}, H^{\infty}$ and $V M O A \cap H^{\infty}$ are examples of spaces which have the $f$-property bur fail to have the $K$-property [35].

The first example of a subspace of $H^{1}$ not possessing the $f$-property is due to Gurarii [33] who proved that the space of analytic functions in $\mathbb{D}$ whose sequence of Taylor coefficients is in $\ell^{1}$ does not have the $f$-property. Anderson [6] proved 
that the space $\mathcal{B}_{0} \cap H^{\infty}$ does not have the $f$-property. Later on it was proved in [29] that if $1 \leq p<\infty$ then $H^{p} \cap \mathcal{B}$ fails to have the $f$-property also.

Since as we have already mentioned the Besov spaces $B^{p}(1<p<\infty)$ and the $Q_{s}$ spaces $(0<s \leq 1)$ have the $K$-property (and, also, the $f$-property), it seems natural to investigate whether the spaces of multipliers and the spaces $Q_{s, \log , \alpha}$ that we have considered in our work have also these properties. We shall prove the following results.

Theorem 4.1. The spaces of multipliers $M\left(B^{p}, Q_{s}\right)(0<s \leq 1,1 \leq p<\infty)$, $M\left(Q_{s_{1}}, Q_{s_{2}}\right) \quad\left(0<s_{1}, s_{2} \leq 1\right)$, and $M\left(B^{p}, B^{q}\right) \quad(1 \leq p, q<\infty)$ have the $f$ property.

Theorem 4.2. For $\alpha>0$ and $0<s<1$ the space $Q_{s, \log , \alpha}$ has the $K$-property.

Theorem 4.1 follows readily from the following result.

Lemma 4.3. Let $X$ and $Y$ be to Banach spaces of analytic functions which are continuously contained in $H^{1}$. Suppose that $X$ contains the constants functions and that $Y$ has the $f$-property. Then the space of multipliers $M(X, Y)$ also has the $f$-property.

Proof. Since $X$ contains the constants functions $M(X, Y) \subset Y \subset H^{1}$.

Suppose that $F \in M(X, Y), I$ is an inner function, and $F / I \in H^{1}$. Take $f \in X$. Then $f F \in Y \subset H^{1}$ and then $f F / I \in H^{1}$. Since $Y$ has the $f$-property, it follows that $f F / I \in Y$. Thus, we have proved that $F / I \in M(X, Y)$.

Theorem 4.2 will follows from a characterization of the spaces $Q_{s, \log , \alpha}$ in terms of pseudoanalytic continuation. We refer to Dyn'kin's paper [24] for similar descriptions of classical smoothness spaces, as well as for other important applications of the pseudoanalytic extension method.

Let, $\mathbb{D}_{-}$denotes the region $\{z \in \mathbb{C}:|z|>1\}$, and write

$$
z^{*} \stackrel{\text { def }}{=} 1 / \bar{z}, \quad z \in \mathbb{C} \backslash\{0\}
$$

We shall need the Cauchy-Riemann operator

$$
\bar{\partial}=\frac{\partial}{\partial \bar{z}} \stackrel{\text { def }}{=} \frac{1}{2}\left(\frac{\partial}{\partial x}+i \frac{\partial}{\partial y}\right), \quad z=x+i y .
$$

The following result is an extension of [23, Theorem 1].

Theorem 4.4. Suppose that $0<s<1, \alpha>0$, and $f \in \cap_{0<q<\infty} H^{q}$. Then the following conditions are equivalent.

(i) $f \in Q_{s, \log , \alpha}$.

(ii) $\sup _{|a|<1}\left(\log \frac{2}{1-|a|}\right)^{2 \alpha} \int_{\mathbb{D}}\left|f^{\prime}(z)\right|^{2}\left(\frac{1}{\left|\varphi_{a}(z)\right|^{2}}-1\right)^{s} d A(z)<\infty$. 
(iii) There exists a function $F \in C^{1}\left(\mathbb{D}_{-}\right)$satisfying

$$
\begin{aligned}
& F(z)=O(1), \quad \text { as } z \rightarrow \infty, \\
& \lim _{r \rightarrow 1^{+}} F\left(r e^{i \theta}\right)=f\left(e^{i \theta}\right), \quad \text { a.e. and in } L^{q}([-\pi, \pi]) \text { for all } q \in[1, \infty), \\
& \sup _{|a|<1}\left(\log \frac{2}{1-|a|}\right)^{2 \alpha} \int_{\mathbb{D}_{-}}|\bar{\partial} F(z)|^{2}\left(\left|\varphi_{a}(z)\right|^{2}-1\right)^{s} d A(z)<\infty .
\end{aligned}
$$

Theorem 4.4 can be proved following the arguments used in the proof of [23, Theorem 1], we omit the details. Once Theorem 4.4 is established, noticing that if $\alpha>0$ and $0<s<1$ then $Q_{s, \log \alpha} \subset Q_{s} \subset B M O A$, Theorem 4.2 can be proved following the steps in the proof of [23, Theorem 2]. Again, we omit the details.

Acknowledgements. We wish to thank the referees for reading carefully the paper and making a number of nice suggestions to improve it.

\section{REFERENCES}

[1] A. Aleman and J. A. Cima, An integral operator on $H^{p}$ and Hardy's inequality, J. Anal. Math. 85 (2001), 157-176.

[2] A. Aleman, P. L. Duren, M. J. Martín and D. Vukotić, Multiplicative isometries and isometric zero-divisors, Canad. J. Math. 62 (2010), no. 5, 961-974.

[3] A. Aleman and A. Simbotin, Estimates in Möbius invariant spaces of analytic functions, Complex Var. Theory Appl. 49 (2004), no. 7-9, 487-510.

[4] A. Aleman and A. G. Siskakis, An integral operator on $H^{p}$, Complex Variables Theory Appl. 28 (1995), no. 2, 149-158.

[5] A. Aleman and A. G. Siskakis, Integration operators on Bergman spaces, Indiana Univ. Math. J. 46 (1997), no. 2, 337-356.

[6] J. M. Anderson, On division by inner factors, Comment. Math. Helv. 54 (1979), no. 2, 309-317.

[7] J. M. Anderson, J. Clunie and Ch. Pommerenke, On Bloch functions and normal functions, J. Reine Angew. Math. 270 (1974), 12-37.

[8] J. Arazy, S. D. Fisher and J. Peetre, Möbius invariant function spaces, J. Reine Angew. Math. 363 (1985), 110-145.

[9] N. Arcozzi, R. Rochberg and E. Sawyer, Carleson measures for analytic Besov spaces. Rev. Mat. Iberoamericana 18 (2002), no. 2, 443-510.

[10] R. Aulaskari and G. Csordas, Besov spaces and the $Q_{q, 0}$ classes, Acta Sci. Math. (Szeged) 60 (1995), no. 1-2, 31-48.

[11] R. Aulaskari, D. Girela and H. Wulan, Taylor coefficients and mean growth of the derivative of $Q_{p}$ functions, J. Math. Anal. Appl. 258 (2001), no. 2, 415-428.

[12] R. Aulaskari and P. Lappan, Criteria for an analytic function to be Bloch and a harmonic or meromorphic function to be normal, Complex Analysis and its Applications (Harlow), Pitman Research Notes in Math, vol. 305, Longman Scientific and Technical, 1994, 136146.

[13] R. Aulaskari, D. A. Stegenga and J. Xiao, Some subclasses of BMOA and their characterization in terms of Carleson measures, Rocky Mountain J. Math. 26 (1996), no. 2, 485-506.

[14] R. Aulaskari, J. Xiao and R. Zhao, On subspaces and subsets of BMOA and UBC, Analysis 15 (1995), no. 2, 101-121.

[15] L. Brown and A. L. Shields, Multipliers and cyclic vectors in the Bloch space, Michigan Math. J. 38 (1991), no. 1, 141-146. 
[16] J. J. Donaire, D. Girela and D. Vukotić, On univalent functions in some Möbius invariant spaces, J. Reine Angew. Math. 553 (2002), 43-72.

[17] J. J. Donaire, D. Girela and D. Vukotić, On the growth and range of functions in Möbius invariant spaces, J. Anal. Math. 112 (2010), 237-260.

[18] P. L. Duren, Theory of $H^{p}$ spaces, Academic Press, New York-London, 1970. Reprint: Dover, Mineola-New York, 2000.

[19] P. L. Duren and A. P. Schuster, Bergman Spaces, Math. Surveys and Monographs, Vol. 100, American Mathematical Society, Providence, Rhode Island, 2004.

[20] K. M. Dyakonov, Factorization of smooth analytic functions via Hilbert-Schmidt operators, (in Russian), Algebra i Analiz 8(1996), no. 4, 1-42. English translation in St. Petersburg Math. J. 8 (1997), no. 4, 543-569.

[21] K. M. Dyakonov, Equivalent norms on Lipschitz-type spaces of holomorphic functions, Acta. Math. 178 (1997), 143-167.

[22] K.M. Dyakonov, Holomorphic functions and quasiconformal mappings with smooth modulii, Adv. in Math. 187 (2004), 146-172.

[23] K. M. Dyakonov and D. Girela, On $Q_{p}$ spaces and pseudoanalytic extension, Ann. Acad. Sci. Fenn. Math. 25 (2000), no. 2, 477-486.

[24] E. M. Dyn'kin, The pseudoanalytic extension, J. Anal. Math. 60 (1993), 45-70.

[25] P. Galanopoulos, D. Girela and M. J. Martín, Besov spaces, multipliers and univalent functions, Complex Anal. Oper. Theory 7 (2013), no. 4, 1081-1116.

[26] P. Galanopoulos, D. Girela and J. A. Peláez, Multipliers and integration operators on Dirichlet spaces, Trans. Amer. Math. Soc. 363 (2011), no. 4, 1855-1886.

[27] D. Girela, Growth of the derivative of bounded analytic functions, Complex Variables Theory Appl. 20 (1992), no. 1-4, 221-227.

[28] D. Girela, Analytic functions of bounded mean oscillation, Complex Function Spaces, Mekrijärvi 1999 Editor: R. Aulaskari. Univ. Joensuu Dept. Math. Rep. Ser. 4, Univ. Joensuu, Joensuu (2001) pp. 61-170.

[29] D. Girela, C. González and J. A. Peláez, Multiplication and division by inner functions in the space of Bloch functions, Proc. Amer. Math. Soc. 134 (2006), no. 5, 1309-1314.

[30] D. Girela and N. Merchán, A generalized Hilbert operator acting on conformally invariant spaces, Banach J. Math. Anal. 12 (2018), no. 2, 374-398.

[31] D. Girela, M. Nowak and P. Waniurski, On the zeros of Bloch functions, Math. Proc. Cambridge Philos. Soc. 129 (2000), no. 1, 117-128.

[32] D. Girela and J. A. Peláez, Carleson measures, multipliers and integration operators for spaces of Dirichlet type, J. Funct. Anal. 241 (2006), no. 1, 334358.

[33] V. P. Gurarii, On the factorization of absolutely convergent Taylor series and Fourier integrals, (in Russian) Zap. Naučn. Sem. Leningrad. Otdel. Mat. Inst. Steklov. (LOMI) 30 (1972), 15-32.

[34] V. P. Havin, On the factorization of analytic functions smooth up to the boundary (Russian), Zap. Naucn. Sem. Leningrad. Otdel. Mat. Inst. Steklov. (LOMI) 22 (1971) 202-205.

[35] H. Hedenmalm, On the $f$ - and $K$-properties of certain function spaces, Contemporary Math. 91 (1989), 89-91.

[36] H. Hedenmalm, B. Korenblum and K. Zhu, Theory of Bergman Spaces, Graduate Texts in Mathematics, Vol. 199, Springer, New York, Berlin, etc. 2000.

[37] F. Holland and D. Walsh, Growth estimates for functions in the Besov spaces $A_{p}$, Proc. Roy. Irish Acad. Sect. A 88 (1988), 1-18.

[38] B. I. Korenblum, A certain extremal property of outer functions, (in Russian), Mat. Zametki 10 (1971), 53-56. English translation in Math. Notes 10 (1971), 456-458.

[39] B. I. Korenblum and V. M. Faŭvyševskiŭ, A certain class of compression operators that are connected with the divisibility of analytic functions, (in Russian), Ukrain. Mat. Z. 24 (1972), 692-695. English translation in Ukrainian Math. J. 24 (1973), 559-561.

[40] J. M. Ortega and J. Fàbrega, Pointwise multipliers and corona type decomposition in BMOA, Ann. Inst. Fourier (Grenoble) 46 (1996), no. 1, 111-137. 
[41] J. Pau and J. A. Peláez, Multipliers of Möbius invariant $Q_{s}$ spaces, Math. Z. 261 (2009), no. $3,545-555$.

[42] J. Pau and J. A. Peláez, On the zeros of functions in Dirichlet-type spaces, Trans. Amer. Math. Soc. 363 (2011), no. 4, 1981-2002.

[43] Ch. Pommerenke, Schlichte Funktionen und analytische Funktionen von beschrnkter mittlerer Oszillation, Comment. Math. Helv. 52 (1977), no. 4, 591-602.

[44] L. E. Rubel and R. M. Timoney, An extremal property of the Bloch space, Proc. Amer. Math. Soc. 75 (1979), no. 1, 45-49.

[45] D. Stegenga, Multipliers of the Dirichlet space. Illinois J. Math. 24 (1980), no. 1, 113-139.

[46] K. Stroethoff, Besov-type characterisations for the Bloch space, Bull. Austral. Math. Soc. 39 (1989), no. 3, 405-420.

[47] R. M. Timoney, Natural function spaces, J. London Math. Soc. (2) 41 (1990), no. 1, 78-88.

[48] S. A. Vinogradov, Multiplication and division in the space of analytic functions with area integrable derivative, and in some related spaces (in russian), Zap. Nauchn. Sem. S.-Peterburg. Otdel. Mat. Inst. Steklov. (POMI) 222 (1995), Issled. po Linein. Oper. i Teor. Funktsii 23, 45-77, 308. English translation in J. Math. Sci. (New York) 87, no. 5 (1997), 3806-3827.

[49] Z. Wu, Carleson measures and multipliers for Dirichlet spaces. J. Funct. Anal. 169 (1999), no. $1,148-163$.

[50] J. Xiao, Carleson measure, atomic decomposition and free interpolation from Bloch space, Ann. Acad. Sci. Fenn. Ser. A I Math. 19 (1994), 35-46.

[51] J. Xiao, The $Q_{p}$ corona theorem, Pacific J. Math. 194 (2000), no. 2, 491-509.

[52] J. Xiao, Holomorphic Q classes, Lecture Notes in Mathematics 1767, Springer-Verlag, 2001.

[53] J. Xiao, Geometric Q functions, Frontiers in Mathematics. Birkhäuser Verlag, 2006.

[54] J. Xiao, The $Q_{p}$ Carleson measure problem, Adv. Math. 217 (2008), no. 5, 2075-2088.

[55] R. Zhao, On logarithmic Carleson measures, Acta Sci. Math. (Szeged) 69 (2003), no. 3-4, 605-618.

[56] K. Zhu, Analytic Besov spaces, J. Math. Anal. Appl. 157 (1991), 318-336.

[57] K. Zhu, A class of Möbius invariant function spaces, Illinois J. Math. 51 (2007), no. 3, 977-1002.

[58] K. Zhu, Operator Theory in Function Spaces, Marcel Dekker, New York, 1990. Reprint: Math. Surveys and Monographs, Vol. 138, American Mathematical Society, Providence, Rhode Island, 2007.

[59] N. Zorboska, Multiplication and Toeplitz operators on the analytic Besov spaces. In: More Progress in Analysis: Proceedings of the 5th International. Isaac Congress. Catania, Italy, 2530 July 2005. Editors: H. G. W. Begehr and F. Nicolosi. World Scientific (2009), pp. 387-396.

[60] A. Zygmund, Trigonometric Series, Vol. I and Vol. II, Second edition, Camb. Univ. Press, Cambridge, 1959.

Departamento de Análisis Matemático, Estadística e Investigación Operativa, y Matemática Aplicada, Universidad de Málaga, 29071 Málaga, Spain

E-mail address: girela@uma.es

Departamento de Matemática Aplicada, Universidad de Málaga, 29071 Málaga, SPAIN

E-mail address: noel@uma.es 